\title{
Câncer de mama na gravidez: diagnóstico e tratamento
}

\author{
Breast cancer in pregnancy: diagnosis and treatment
}

\author{
Cancer de mama en el embarazo: diagnóstico y tratamiento
}

\author{
Denise L. M. Monteiro," Daniela C. S. Menezes, Camila L. Nunes, \\ Clara A. Antunes, Erica M. de Almeida, Alexandre J. B. Trajano
}

\begin{abstract}
Resumo
O objetivo do estudo é avaliar o diagnóstico e a terapêutica do câncer de mama em gestantes, visto que a gravidez pode potencialmente gerar retardo na abordagem da doença e, consequentemente, pior prognóstico do que na mulher não gestante. Associa-se ainda o baixo índice de suspeição da doença neste período e relutância de médicos e pacientes em realizar testes radiográficos e procedimentos invasivos. O câncer de mama é a neoplasia maligna feminina mais frequente. Sua incidência na gestação é baixa, variando de 1:3000 a 1:10.000 gravidezes, apresentando-se geralmente em estágio avançado durante a gravidez. A condução do caso frequentemente gera dificuldades e angústia para a gestante, sua família e para os profissionais de saúde envolvidos, em função do dilema criado entre a terapia ideal para a mãe e o bem-estar do feto. O diagnóstico baseia-se no teste triplo que consiste em exame clínico, imagem e biópsia, visando avaliar a extensão da doença na mama e nos linfonodos, identificar múltiplos focos ou eventual tumor bilateral, bem como prevenir metástases sistêmicas e controlar localmente a doença. O adiamento da terapêutica, a fim de proteger o concepto, pode comprometer a saúde materna. A mastectomia radical modificada e a radical clássica podem ser utilizadas com segurança. Deve-se realizar o esvaziamento axilar, pois metástases são comumente encontradas na gravidez e o comprometimento linfonodal influencia na escolha da quimioterapia. Evidências sugerem segurança e eficácia do esquema 5-fluorouracil, doxorrubicina (ou epirrubicina) e ciclofosfamida durante o segundo e o terceiro trimestres da gestação. A radioterapia deve ser adiada, sempre que possível, para depois do parto. Este estudo visa ainda incentivar o exame das mamas durante o exame ginecológico de rotina e pré-natal, conscientizando paciente e equipe de saúde de sua importância no ciclo grávido-puerperal.
\end{abstract}

Descritores: Câncer de mama; Gravidez; Diagnóstico; Tratamento.

\begin{abstract}
The aim of the study is to evaluate diagnosis and treatment of breast cancer in pregnant women, since pregnancy can potentially generate delay in disease approach and consequently worst prognosis than in non-pregnant women. There is also a low index of suspicion of the disease in this period and reluctance of patients and physicians to perform radiographic tests and invasive procedures. Breast cancer is the most common female malignancy. Its incidence in pregnancy is low, ranging from 1:3000 to 1:10,000 pregnancies, usually presenting at an advanced stage during pregnancy. The disease management often generates difficulties and distress for the mother, her family and health professionals involved, depending on the dilemma created between the ideal therapy for the mother and fetus welfare. Diagnostic approach is based on triple test consisting of clinical, imaging and biopsy examination to evaluate disease extension in breast and lymph nodes, identify possible multiple foci or bilateral tumors, and prevent systemic metastasis and manage local disease. Therapy delay in order to protect fetus can compromise maternal health. Modified radical mastectomy and classic radical mastectomy can be safely used. Axillary dissection should be performed, since metastases are commonly found in pregnancy and lymph node involvement defines chemotherapy. Evidence suggests safety and efficacy of 5-fluorouracil, doxorubicin (or epirubicin) and cyclophosphamide during the second and third trimester of pregnancy. Radiotherapy should be postponed whenever possible after delivery. This study also aims to encourage breast examination during routine gynecologic evaluation and prenatal care, making patient and health team aware of its importance during pregnancy and childbirth.
\end{abstract}

Keywords: Breast Cancer; Pregnancy; Diagnosis; Treatment. 


\section{Resumen}

El objetivo del estudio es evaluar el diagnóstico y la terapéutica del cáncer de mama en gestantes, ya que el embarazo puede potencialmente producir retraso en el abordaje de la enfermedad y, consecuentemente, peor pronóstico que en la mujer no gestante. Se asocia el bajo índice de sospecha de la enfermedad en este periodo y la resistencia de médicos y pacientes a realizar tests radiográficos y procedimientos invasivos. El cáncer de mama es la neoplasia femenina más frecuente. Su incidencia en la gestación es baja, variando de 1:3000 a 1:10.000 embarazos, presentándose generalmente en estadio avanzado durante el embarazo. El manejo del caso frecuentemente genera dificultades para la gestante, su familia y para los profesionales de salud involucrados en el dilema creado entre la terapia ideal para la madre y el bien estar del feto. El diagnóstico se basa en el test triple que consiste en el examen clínico, imagen y biopsia, pretendiendo evaluar la extensión de la enfermedad en la mama y en los linfonodos, identificar múltiples focos o un eventual tumor bilateral, así como prevenir metástasis sistémica y controlar localmente la enfermedad. El avance en la terapéutica, a fin de proteger el producto, puede comprometer la salud materna. La mastectomía radical modificada y la radical clásica pueden ser utilizadas con seguridad. Se debe realizar el vaciamiento axilar, pues las metástasis son comúnmente encontradas en el embarazo y el compromiso linfonodal influencia en la elección de la quimioterapia. Evidencias sugieren seguridad y eficacia del esquema 5-fluorouracil, doxorrubicina (o epirrubicina) y ciclofosfamida durante el segundo y el tercer trimestre de la gestación. La radioterapia debe ser retrasada para después del parto. Este estudio pretende incentivar el examen de las mamas durante el examen ginecológico de rutina y prenatal, concientizando a la paciente y equipo de salud sobre su importancia en el ciclo grávido puerperal.

Palabras clave: Cáncer de mama; Embarazo; Diagnóstico; Tratamiento.

\section{Introdução}

Câncer de mama associado à gravidez é definido como aquele diagnosticado durante a gestação, lactação ou no primeiro ano após o parto. É uma situação clínica rara e desafiadora, com incidência variando de 1:3000 a 1:10.000 gestações. ${ }^{1,2,3}$

No Brasil, as taxas de mortalidade por câncer de mama continuam elevadas, provavelmente pelo fato de a doença ainda ser diagnosticada em estádios avançados. Este retardo no diagnóstico acentua-se ainda mais no período gravídico-puerperal, uma vez que as modificações fisiológicas mamárias dificultam a identificação do nódulo suspeito, além do baixo índice de suspeição da doença neste período e relutância de médicos e pacientes em realizar testes radiográficos e procedimentos invasivos neste período., ${ }^{2,45}$

O tratamento precisa ser cuidadosamente instituído, visando a terapia ideal para a mãe portadora do câncer e o bem-estar fetal. $\mathrm{O}$ adiamento da terapêutica, a fim de proteger o concepto, pode comprometer a saúde materna. ${ }^{3}$

O objetivo do estudo é ressaltar a importância do diagnóstico e do tratamento do câncer de mama na gravidez, visto que está sujeito a potenciais causas de retardo e pior prognóstico do que na mulher não gestante. Esta discussão também deve subsidiar o incentivo ao exame das mamas durante o exame ginecológico de rotina e pré-natal, conscientizando paciente e equipe de saúde da importância do exame da mama no ciclo grávido-puerperal.

\section{Diagnóstico do câncer de mama durante a gravidez}

A abordagem diagnóstica baseia-se no teste triplo que consiste em exame clínico, imagem e biópsia. Qualquer massa suspeita encontrada durante a gravidez deve ser investigada. ${ }^{6,8,9}$

O exame clínico das mamas é realizado na consulta obstétrica no primeiro trimestre. Uma vez identificado o nódulo suspeito, este deverá ser investigado por método de imagem. $\mathrm{Na}$ gravidez, o primeiro exame a ser solicitado é a ultrassonografia mamária (USG), devido a seu baixo custo e capacidade de distinguir lesões císticas de sólidas. ${ }^{6-10}$ Os nódulos com bordas irregulares, ecos internos heterogêneos, áreas anecoicas, reforço acústico posterior e distorção da arquitetura mamária merecem estudo anatomopatológico imediato. ${ }^{9,10}$ Durante a gestação e lactação, a identificação de lesões à mamografia é dificultada pela maior densidade do parênquima, intensa vascularização e aumento da celularidade. ${ }^{2,6,7}$ Vale ressaltar que a dose de radiação, com proteção abdominal adequada, fica em torno de 0,004 cGy, sendo insignificante o risco fetal., ${ }^{2,6,8}$ 
A positividade de $90 \%$ em gestantes com câncer de mama invasivo demonstra o valor do método. A mamografia digital é preferida por apresentar maior sensibilidade em mamas densas e redução de $75 \%$ na dose da radiação. ${ }^{8} \mathrm{O}$ exame é útil para avaliar a extensão da doença na mama e em linfonodos, além de identificar múltiplos focos ou eventual tumor bilateral, sendo especialmente relevante na presença de microcalcificações. ${ }^{7,8,11}$ Em lactantes, está indicado o esvaziamento das mamas imediatamente antes da realização do exame. ${ }^{8,11}$

A biópsia constitui o padrão-ouro para o diagnóstico. A punção aspirativa com agulha fina pode ser utilizada para o diagnóstico da massa mamária, mas a core-biópsia ou a biópsia excisional é necessária para o diagnóstico definitivo de câncer invasivo. . $^{3,14}$

Após o estabelecimento do diagnóstico, é necessária pesquisa de metástases ósseas, pulmonares, pleurais, hepáticas ou cerebrais, para o correto estadiamento e tratamento. Não há contraindicações para a realização de radiografia de tórax (0,008 cGy), crânio ou ossos longos (0,001 cGy). A ressonância magnética consiste em potencial ferramenta, sendo ideal para confirmar o diagnóstico clínico ou radiológico das metástases. ${ }^{6,10}$

\section{Conduta na gravidez}

O objetivo do tratamento da gestante com câncer de mama é o mesmo da paciente não grávida: prevenção de metástases sistêmicas e controle local da doença. No entanto, a forma de tratamento precisa ser cuidadosamente avaliada em função dos potenciais efeitos adversos para o feto. ${ }^{3}$

\section{Tratamento cirúrgico}

Razoavelmente seguro é o tratamento definitivo do carcinoma de mama em gestantes. Os riscos da cirurgia durante a gravidez são aborto espontâneo e parto prematuro, não havendo aumento do risco de malformações congênitas. ${ }^{15}$

A excisão ampla, a mastectomia radical modificada e a mastectomia radical clássica podem ser utilizadas com segurança quando não há doença metastática. ${ }^{16} \mathrm{O}$ esvaziamento axilar é preferido, uma vez que metástases são comumente encontradas na gravidez e o comprometimento linfonodal influencia na escolha da quimioterapia. ${ }^{17}$ A mastectomia elimina a necessidade de radioterapia adjuvante e, consequentemente, seus riscos para o feto. A biópsia do linfonodo sentinela parece ser segura na gestação. A utilização do radioisótopo (99 m-Tc) não expõe o feto à quantidade de radiação significativa e, portanto, pode ser utilizada. ${ }^{16,18,19}$

A terapia cirúrgica de conservação mamária (tumorectomia) associada à radioterapia ou quimioterapia neoadjuvante é uma opção para mulheres diagnosticadas em fases avançadas da gestação.,

\section{Quimioterapia (QT)}

A exposição do feto a essa terapia quando administrada em mulheres no segundo ou terceiro trimestre, período posterior à organogênese, resulta em nascidos vivos com baixa morbidade e não parece causar aumento do risco de malformações congênitas. Contudo, pode ser associado ao retardo do crescimento intrauterino e baixo peso ao nascer. ${ }^{3,17}$

Taxanos - grupo de medicamentos antineoplásicos de ação antimitótica, com dados de segurança e eficácia limitados, necessitando mais estudos que avaliem a farmacocinética e a passagem transplacentária. Entretanto, parecem melhorar o prognóstico de mulheres com câncer de mama, particularmente as que apresentam acometimento linfonodal. Os mais usados são o docetaxel (D) e paclitaxel (P). ${ }^{20}$

Trastuzumabe - é um anticorpo monoclonal dirigido ao receptor do fator de crescimento epidermal humano 2 (HER2). Contudo, seu uso na gravidez é contraindicado pelos riscos de oligodramnia ou adramnia, anormalidades esqueléticas, lesão renal, hipoplasia pulmonar e morte neonatal..$^{19,21}$

Lapatinib - afeta tanto HER2/neu (erb B-2) quanto o receptor do fator de crescimento epidérmico (EGFR, também chamado de erbB-1). Foi aprovado para o uso em caso de superexpressão de HER2 em câncer de mama avançados que tenham progredido após exposição ao trastuzumabe. Não é tratamento padrão para câncer de mama em estágio inicial e seu uso não é indicado durante a gravidez e a lactação. ${ }^{3}$

Metotrexato - deve ser evitado em todas as 
fases da gravidez por causa do seu efeito abortivo e teratogênico potencial. ${ }^{22}$

Antraciclinas - o esquema mais utilizado em grávidas com câncer de mama é o esquema FAC: 5-fluorouracil, doxorrubicina (ou epirrubicina) e ciclofosfamida. Evidências sugerem segurança e eficácia do uso das antraciclinas durante o segundo e o terceiro trimestres da gestação, com nível de evidência 2B (Oxford Level of Evidence [LOE] 2B). ${ }^{23} \mathrm{O}$ uso da QT no primeiro trimestre está associado a malformações em mãos e pés, micrognatia e ventriculomegalia entre 10 a $20 \%$ dos fetos. ${ }^{24}$ Portanto, o tratamento quimioterápico não deve ser protelado. No entanto, é recomendável evitar a quimioterapia de três a quatro semanas antes do parto para prevenir a mielossupressão transitória neonatal e potenciais complicações da sepse, além da morte. ${ }^{23}$

\section{Radioterapia}

É utilizada para melhorar a sobrevida de muIheres de alto risco tratadas com mastectomia, otimizando o controle local da doença. ${ }^{25}$ Seu uso deve ser adiado sempre que possível para depois do parto, pois o efeito dessa terapia sobre o concepto depende tanto da dose empregada quanto da idade gestacional, podendo variar de morte do blastocisto e abortamento até retardo de crescimento, microcefalia, lesões oculares e alterações comportamentais e cognitivas., 3,1725

\section{Terapia endócrina}

O tamoxifeno é um modulador seletivo dos receptores de estrogênio. Devido à sua potente ação antiestrogênica, tornou-se parte importante na terapia adjuvante de mulheres com câncer de mama. Entretanto, durante a gravidez, o tamoxifeno e seus metabólitos interagem com os tecidos fetais e embrionários em desenvolvimento. Os estudos em animais demonstram associação com múltiplas malformações e perda fetal. Os dados em humanos, por razões óbvias, são provenientes de estudos retrospectivos, e não baseados em estudos controlados. A relativa alta incidência de malformações associadas, abortamentos e perdas fetais sugere que o uso desta medicação durante a gravidez deve ser impedido. As principais malformações encontradas são genitália ambígua, hipertrofia de clitóris e fenda palatina. ${ }^{26}$

\section{Lactação}

A lactação parece ser segura e possível. O aleitamento na mama contralateral não é afetado. Em casos de cirurgia conservadora e radioterapia, a produção de leite na mama tratada poderá ser menor. Alguns fatores, como incisão próxima a aréola e mamilo, localização do tumor, além de dose e tipo de radiação, podem alterar a produção de leite. De qualquer forma, o aleitamento na mama irradiada não é recomendado devido ao risco da mulher desenvolver mastite, que, caso ocorra, será de manejo delicado. ${ }^{27,28}$

A maioria das drogas (principalmente, os agentes alquilantes) é excretada no leite humano. De forma geral, a lactação deve ser evitada durante a quimioterapia, uso de transtuzumab, lapatinib e terapia endócrina. Não há evidências de que o aleitamento em mulheres com câncer de mama tratado modifique o prognóstico materno. ${ }^{28}$

Apesar de informações fundamentadas em evidências limitadas, o tratamento de câncer durante a gravidez pode ter sucesso, fornecendo para esta população a melhora do prognóstico materno e fetal.

\section{Referências}

1. Brasil. Ministério da Saúde. Instituto Nacional do Câncer. Câncer de Mama. Disponível em: http:// www2.inca.gov.br/wps/wcm/connect/tiposdecancer/ site/home/mama. Acesso em: 22 Abr 2013.

2. Keinan-Boker L, Lerner-Geva L, Kaufman B, Meirow D. Pregnancy-Associated Breast Cancer. IMAJ. 2008;10(10):722-7.

3. Loibl S, Minckwitz GV, Gwyn K, Ellis P, Blohmer JU, Schlegelberger B, M, et al. Breast carcinoma during pregnancy. International recommendations from an expert meeting. Cancer. 2006;106(2):237-46.

4. Antonelli NM, Dotters DJ, Katz VL, Kuller JA. Cancer in pregnancy: a review of the literature: part I. Obstet Gynecol Surv. 1996;51(2):125-34.

5. Jacobs A, Chang CK, Saltl Gl. Coexistence of pregnancy and cancer. Am Surg. 2004;70(11):1025-9.

6. Michael H, Pierre L V, Diane P. Breast cancer, pregnancy and breastfeeding. SOCG Clinical Practice Guidelines. 2002;111:4-5.

7. ROCG Green top guideline. Pregnancy and Breast Cancer. 2004; 2:2-3.

8. Saunders C, Taylor D, Ives A. The role of breast imaging during pregnancy and lactation in the diagnosis of breast malignancy. The Radiographer. 2012;59(4):119-23. 
9. Calderon-Margalit R, Paltiel O. Prevention of breast cancer in women who carry BRCA1 or BRCA2 mutations: a critical review of the literature. Int J Cancer. 2004;112(3): 357-64.

10. Psyrri A, Burtness B. Pregnancy-associated breast cancer. Cancer J. 2005;11(2):83-95.

11. Shah E, Saunders C. Breast Cancer and pregnancy. In: Barnea ER, Jauniaux E, Schwarts PE, editors. Cancer and Pregnancy. London: Springer-Verlag; 2001. p.21-32.

12. Newman LA, Kuerer HM. Advances in breast conservation therapy. J Clin Oncol. 2005;23(8):1685-97.

13. Novotny DB, Maygarden SJ, Shermer RW, Frable WJ. Fine needle aspiration of benign and malignant breast masses associated with pregnancy. Acta Cytol. 1991;35(6):676-86.

14. Gupta RK. The diagnostic impact of aspiration cytodiagnosis of breast masses in association with pregnancy and lactation with an emphasis on clinical decision making. Breast J. 1997;3:131-4.

15. Petrek J. Breast cancer during pregnancy. Cancer. 1994;74(suppl 1):518-27.

16. Rosekranz KM, Lucci A. Surgical treatment of pregnancy associated breast cancer. Breast Dis. 2005-2006;23:87-93.

17. Woo JC, Yu T, Hurd TC. Breast cancer in pregnancy: a literature review. Arch Surg. 2003;138(1):91-8.

18. Mondi MM, Cuenca RE, Ollila DW. Sentinel Lymph node biopsy during pregnancy: Initial clinical experience. Ann Surg Oncol. 2007;14(1):218-21.

19. Spanheimer PM, Graham MM, Sugg SL, ScottConner CE, Weigel RJ. Measurement of uterine radiation exposure from lymphoscintigraphy indicates safety of sentinel lymph node biopsy during pregnancy. Ann Surg Oncol. 2009;16(5):1143-7. http://dx.doi.org/10.1245/s10434-009-0390-z

20. Mir O, Berveiller P, Goffinet F, Treluyer JM, Serreau R, Goldwasser F, et al. Taxanes for breast cancer during pregnancy: a systematic review. Ann Oncol. 2010;21(2):425-6. http://dx.doi.org/10.1093/annonc/mdp517.

21. Romond EH, Perez EA, Bryant J, Suman VJ, Geyer CE Jr, Davidson NE, et al. Trastuzumab plus adjuvant chermotherapy for operable HER2-positive breast cancer. N Engl J Med. 2005;353(16):1673-84.

22. Kelly H, Graham M, Humes E, Dorflinger LJ, Boggess KA, O'Neil BH, et al. Delivery of a healthy baby after first-trimester maternal exposure to lapatinib. Clin Breast Cancer. 2006;7(4):339-41.

23. Doll DC, Ringenberg QS, Yarbro JW. Antineoplastic agents and pregnancy. Semin Oncol. 1989;16(5):337-46.

24. Leyder M, Laubach M, Breugelmans M, Keymolen K, De Greve J, Foulon W. Specific congenital malformations after exposure to cyclophosphamide epirubicin and 5-fluorouracil during the first trimester of pregnancy. Gynecol Obstet Invest. 2011;71(2):141-4. http://dx.doi.org/10.1159/000317264
25. Kal HB, Struikmans H. Radiotherapy during pregnancy: fact and fiction. Lancet Oncol. 2005; 6(5):328-33.

26. Braems G, Denys H, De Wever O, Cocquyt V, Van den Broecke R. Use of tamoxifen before and during pregnancy. Oncologist. 2011;16(11):1547-51. http:// dx.doi.org/10.1634/theoncologist.2011-0121

27. Moran MS, Colasanto JM, Haffty BG, Wilson LD, Lund MW, Higgins SA. Effects of breast-conserving therapy on lactation after pregnancy. Cancer J. 2005;11(5):399-403.

28. Azim HA Jr, Bellettini G, Gelber S, Peccatori FA. Breast-feeding after breast cancer: if you wish, madam. Breast Cancer Res Treat. 2009;114(1):7-12.

\section{Denise L. M. Monteiro}

Disciplina de Obstetrícia. Departamento de Ginecologia e Obstetrícia. Centro Universitário Serra dos Órgãos (UNIFESO). Teresópolis, RJ, Brasil.

Disciplina de Obstetrícia. Departamento de Ginecologia e Obstetrícia. Faculdade de Ciências Médicas da Universidade do Estado do Rio de Janeiro. Rio de Janeiro, RJ, Brasil.

\section{Daniela C. S. Menezes}

Departamento Ginecologia e Obstetrícia. Faculdade de Ciências Médicas. Universidade do Estado do Rio de Janeiro. Rio de Janeiro, RJ, Brasil.

\section{Camila L. Nunes}

Iniciação Científica, FAPERJ. Faculdade de Ciências Médicas. Universidade do Estado do Rio de Janeiro, Rio de Janeiro, RJ, Brasil.

\section{Clara A. Antunes}

Programa de Residência Médica em Ginecologia e Obstetrícia. Hospital Universitário Pedro Ernesto. Universidade do Estado do Rio de Janeiro. Rio de Janeiro, RJ, Brasil.

\section{Erica M. de Almeida}

Programa de Residência Médica em Ginecologia e Obstetrícia. Hospital Universitário Pedro Ernesto. Universidade do Estado do Rio de Janeiro. Rio de Janeiro, RJ, Brasil.

\section{Alexandre J. B. Trajano}

Departamento de Ginecologia e Obstetrícia. Faculdade de Ciências Médicas. Universidade do Estado do Rio de Janeiro. Rio de Janeiro, RJ, Brasil.

Departamento de Ginecologia e Obstetrícia. Faculdade de Medicina. Universidade Unigranrio. Rio de Janeiro, RJ, Brasil 